\title{
Auger de-excitation of metastable molecules at metallic surfaces
}

\author{
Johannes Marbach* Franz Xaver Bronold, and Holger Fehske \\ Institut für Physik, Ernst-Moritz-Arndt-Universität Greifswald, 17489 Greifswald, Germany
}

(Dated: August 10, 2018)

\begin{abstract}
We study secondary electron emission from metallic surfaces due to Auger de-excitation of diatomic metastable molecules. Our approach is based on an effective model for the two active electrons involved in the process - a molecular electron described by a linear combination of atomic orbitals when it is bound and a two-center Coulomb wave when it is not and a metal electron described by the eigenfunctions of a step potential - and employs Keldysh Green's functions. Solving the Dyson equation for the retarded Green's function by exponential resummation we are able to treat time-nonlocal self-energies and to avoid the wide-band approximation. Results are presented for the de-excitation of $N_{2}\left({ }^{3} \Sigma_{u}^{+}\right)$on aluminum and tungsten and discussed in view of previous experimental and theoretical investigations. We find quantitative agreement with experimental data for tungsten indicating that the effective model captures the physics of the process quite well. For aluminum we predict secondary electron emission due to Auger de-excitation to be one to two orders of magnitude smaller than the one found for resonant charge-transfer and subsequent auto-detachment.
\end{abstract}

PACS numbers: 34.35.+a, 79.20.Rf, 79.20.Hx

\section{INTRODUCTION}

De-excitation of metastable atoms and molecules with simultaneous release of an electron is a surface scattering process of great technological importance. Deexcitation of atoms is used as a surface-sensitive electron spectroscopy ${ }^{1-4}$ and de-excitation of molecules is an important process in molecular low-temperature gas discharges. It is one of the main wall-based secondary electron emission channels controlling, together with wall recombination and various volume-based charge production and destruction channels, the overall charge balance in the discharge.$^{\frac{5}{5}}$ In the de-excitation process both the target and the projectile are composite objects. A great variety of reaction channels is thus conceivable making the investigation of this scattering process a challenging task, particularly for molecules.

Stracke et al. 6 experimentally investigated the deexcitation of metastable nitrogen $N_{2}\left({ }^{3} \Sigma_{u}^{+}\right)$molecules on a tungsten surface and proposed two main reaction channels. Firstly, the Auger de-excitation (also referred to as Penning de-excitation),

$$
N_{2}\left({ }^{3} \Sigma_{u}^{+}\right)+e_{m} \rightarrow N_{2}\left({ }^{1} \Sigma_{g}^{+}\right)+e_{f},
$$

where $e_{m}$ and $e_{f}$ denote an electron inside the metal and a free electron, respectively, and secondly, the formation of the $N_{2}^{-}\left({ }^{2} \Pi_{g}\right)$ shape resonance with subsequent autodetachment,

$$
N_{2}\left({ }^{3} \Sigma_{u}^{+}\right)+e_{m} \rightarrow N_{2}^{-}\left({ }^{2} \Pi_{g}\right) \rightarrow N_{2}\left({ }^{1} \Sigma_{g}^{+}\right)+e_{f} .
$$

Stracke et al. 6 concluded that out of these two competing processes reaction (2) should be more efficient, as it is a combination of two single-electron charge-transfer transitions, whereas (1) represents a less probable twoelectron transition. Using thermal molecules they measured the energy spectrum of the released electron and estimated the overall secondary electron emission coefficient $\gamma_{e}$, that is, the probability for releasing an electron by a single metastable molecule de-excitation at the surface, to be about $10^{-3}-10^{-2}$. The experimental estimate for $\gamma_{e}$ does not discriminate between the two reaction channels. It rather includes both channels. Indeed, Stracke et al. 6 mention that in the spectrum of the emitted electron they also observe a weak signal due to Auger de-excitation. It is one order of magnitude weaker than the signal due to charge-transfer.

Based on the assumption that the charge-transfer channel (2) is the dominant one Lorente et al. ${ }^{7}$ theoretically investigated the de-excitation of $N_{2}\left({ }^{3} \Sigma_{u}^{+}\right)$molecules on an aluminum surface. The resonance-driven secondary electron emission coefficient resulting from their calculated electron emission spectrum is about $10^{-1}$ which is one order of magnitude larger than the value Stracke et al. 6 give for tungsten. The Penning channel (11) was not considered by Lorente and coworkers $\stackrel{7}{-}$ Its strength for an aluminum surface is thus unknown.

In the present work we adopt the point of view complementary to Lorente et al.' $s \frac{7}{7}$ investigating Penning deexcitation while neglecting any contribution due to resonant charge-transfer. In particular for tungsten the efficiency of the Penning process may be comparable to the efficiency of the charge-transfer process, because the molecular orbital hosting the hole in the electronic configuration of $N_{2}\left({ }^{3} \Sigma_{u}^{+}\right)$is roughly $2.5 \mathrm{eV}$ below the bottom of the conduction band of tungsten $\frac{8,9}{}$ To bring this orbital in resonance with conduction band states of the metal requires therefore a large image shift and broadening due to the interaction with the metal. Rough estimates of these two effects based on what is known about them for alkali atoms interacting with surfaces ${ }^{10}$ imply that the resonance condition can only be met for vibrationally excited $N_{2}\left({ }^{3} \Sigma_{u}^{+}\right)$states. Thus, at least for $N_{2}\left({ }^{3} \Sigma_{u}^{+}\right)$in its vibrational ground state, Penning de-excitation and chargetransfer are eye-to-eye competitors. For aluminum the electronic band structure is much more favorable for the charge-transfer scenario ${ }^{11}$ Here, the bottom of the con- 
duction band is only $1 \mathrm{eV}$ above the molecular orbital in question. This energy difference may be bridged by the combined action of image shift and level broadening.

In order to theoretically analyze the Auger deexcitation of diatomic molecules on metallic surfaces we adopt an effective two-electron model, where one electron resides in the conduction band of the metal and the other in the excited state of the molecule. The metal is modeled as a half-space containing a free electron gas characterized by a work function and a conduction band depth, while the molecule is modeled in terms of a two-level system with energy spacing corresponding to the excitation energy of the metastable state. Focusing on the situation where the emitted electron (Auger electron) originates from the molecule we describe it by a two-center Coulomb wave. The coupling between the molecule and the metal is through the Coulomb interaction between a metal electron and the electron in the excited state of the molecule. Image interactions are neglected (except for the emitted electron, where we include it in terms of a surface transmission function) because they give rise to a hybridization of metallic and molecular single-electron states $\frac{10}{10}$ which we prefer to discuss in connection with direct charge-transfer.

Although our model for Auger de-excitation is somewhat crude, it contains the most relevant degrees of freedom in a reasonable approximation and can be parameterized by energies which are relatively easy to obtain. In particular the latter aspect is rather important for us, because our interest in Auger de-excitation and related processes stems from their relevance for bounded lowtemperature gas discharges. It is well known that electrons can be released from plasma walls by de-excitation of metastable species but for most wall materials and projectiles the secondary electron emission coefficient is unknown. A flexible, easy-to-use microscopic model for its calculation is thus needed.

Following the lead of Makoshi and coworkers, $\stackrel{12.13}{,}$ who investigated the de-excitation of metastable atoms, we employ in the following the Keldysh technique $\frac{14-17}{17}$ to calculate within the trajectory approximation $\underline{18}$ the secondary electron emission coefficient and the spectrum of the emitted electron for a diatomic metastable molecule hitting a metallic surface. A description of this type of surface collision with Green's functions $\frac{12,13,19-22}{2}$ is mathematically more demanding than using rate equations ${ }^{18,23}-27$ Green's functions are however rather flexible in handling the non-adiabaticity of the projectile's motion, $\underline{12,13}$ the Coulomb correlations on the projectile,,$\frac{19-21}{2}$ and the collective electronic excitations of the surface ${ }^{22}$ In addition, image shifts and level broadening due to image interactions as well as vibrations of the molecule may also be included in a theoretical description based on Green's functions.

In contrast to Makoshi's work, $, 12,13$ our approach is not restricted to time-local Auger self-energies and thus to the wide-band approximation. To overcome this limitation we solve the Dyson equation for the retarded Green's function by exponential resummation ${ }^{28}$ Our approach is also not restricted to phenomenological Auger matrix elements. We work with the full matrix element, exploiting only the locality of the bound molecular states and the large distance of the molecule's turning point from the surface. Although the final equations for the secondary electron emission coefficient and the spectrum of the Auger electron are highly complex they can be numerically evaluated within an interpolatory grid-based Monte-Carlo integration scheme.

We specifically apply our approach to Auger deexcitation of $N_{2}\left({ }^{3} \Sigma_{u}^{+}\right)$on an aluminum or a tungsten surface. The metastable molecule is assumed to be in its vibrational ground state and the molecule's turning point is obtained from the surface potential applicable to $N_{2}\left({ }^{3} \Sigma_{u}^{+}\right)$on metallic surfaces. For an aluminum surface we find the secondary electron emission coefficient due to Auger de-excitation (11) to be one to two orders of magnitude smaller than the one deduced from Lorente et al.' 's 7 theoretical study of the direct charge-transfer channel (2) and for tungsten we find good quantitative agreement with an experimental estimate based on Stracke et al. 's $\mathbf{6}^{6}$ measurements.

The paper is structured in the following manner. In the next section we give more details about the effective model on which our analysis of Auger de-excitation is based. In Sect. III we employ the Keldysh technique to extract physical quantities from the model. Thereafter we describe in Sec. IV] the numerical scheme we employed for the calculation of the secondary electron emission coefficient and the energy spectrum of the Auger electron. Results are presented in Sec. $\mathrm{V}$. We conclude the paper in Sec. VI and complement it by two appendices. Appendix A contains the explicit form of the wave functions we used in our calculations and Appendix B fixes the notations of the Keldysh formalism.

\section{MODEL}

We investigate the de-excitation of a metastable nitrogen molecule impacting on a metallic surface with simultaneous release of an electron. The model we employ is an effective one that concentrates on the most important degrees of freedom and enables us to describe the system by a few parameters which are accessible through experiments or theoretical calculations. The primary goal will be to calculate the secondary electron emission coefficient $\gamma_{e}$.

Focusing on the essentials of the process, we introduce from the start some simplifications and restrictions. First, we assume the metal surface to be planar, ideal, uncharged, and to stretch over the entire half space $z<0$. Furthermore, we consider only the dominant metastable state $N_{2}\left({ }^{3} \Sigma_{u}^{+}\right)$. In addition, we employ the trajectory approximation, 23 that is, we decouple the translational motion of the molecule from the dynamics of the system and externally supply its trajectory. Finally, the 


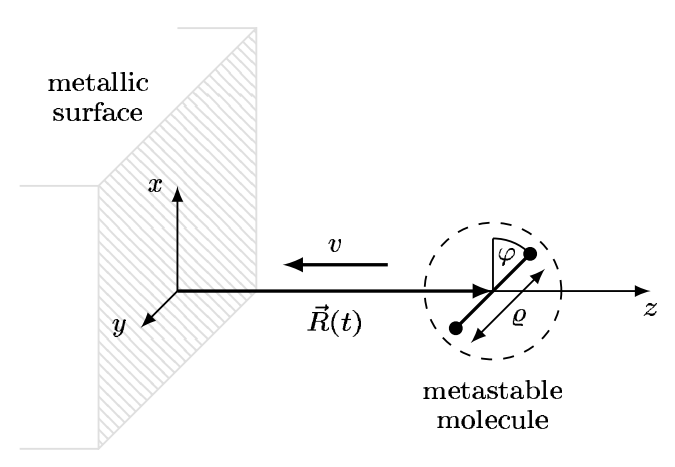

FIG. 1: Schematic illustration of the collision geometry.

molecule is assumed to impact the surface under normal incidence with constant velocity $v$ and constant angle $\varphi$ of its axis to the surface. Because of the translational symmetry of the solid surface in the $x$-y-plane, it is then sufficient to consider only rotations of the molecule axis about one particular axis in this plane, for instance, the $y$-axis (see Fig 1).

We now cast the model assumptions into mathematical form, starting with the trajectory. Assuming the molecule to start moving at $t_{0}=-\infty$ and to hit the surface at $t=0$ the trajectory of its center of mass is

$$
\vec{R}(t)=z_{R}(t) \vec{e}_{z}=\left(v|t|+z_{0}\right) \vec{e}_{z},
$$

where $z_{0}$ denotes the turning point. The center of mass motion is classical. Hence, the turning point $z_{0}$ can be determined by considering the motion of the molecule in the molecule-surface interaction potential $V_{S}(z)$ for given initial kinetic energy $\varepsilon_{k i n}$. Using a Morse-type potential, energy conservation gives for the position of the turning point

$$
z_{0}=z_{e}-\frac{1}{a} \ln \left(1+\sqrt{1+\frac{\varepsilon_{k i n}}{d}}\right)
$$

with material parameters $d, a$, and $z_{e}{ }^{29}$ For metals

$$
d=0.738 \mathrm{eV}, \quad a=640 \mathrm{pm}^{-1}, \quad z_{e}=245 \mathrm{pm} .
$$

According to the authors of Ref. 29 these values are not very specific to the particular metal.

To set-up, for a given trajectory, a Hamiltonian for a nitrogen molecule de-exciting at a metal surface we follow Cazalilla et al. ${ }^{27}$ and distinguish between indirect de-excitation (Penning process, solid lines in Fig. 2), in which the electron is emitted from the molecule, and direct de-excitation (exchange process, dashed lines in Fig. (2), in which the electron is emitted from the surface. It must be stressed that although the asymptotic form of the wave function of the emitted electron is in both cases a plane wave, in the region relevant for the calculation of the matrix element, the Auger wave function for the Penning process resembles a single-electron continuum wave function of the nitrogen molecule whereas the Auger wave function for the exchange process is basically a continuum state of the solid with positive energy. In the following we will only consider the Penning process because our calculations showed that its matrix element is much larger than the matrix element associated with the exchange process, a consequence of the orthogonality of the bound molecular wave functions.

We construct the Hamiltonian by combining three different kinds of single-electron states to a single-electron basis: the single-electron states of the conduction band of the solid surface, which we approximate by the states corresponding to an electron trapped by a step potential

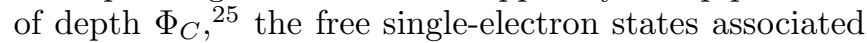
with the molecule's continuum for which we use a twocenter Coulomb wave, $\stackrel{30-32}{=}$ and effective single-electron states for the bound states of the molecule. To keep the description of the molecule as simple as possible we approximate the latter by a degenerate two-level system keeping, within the LCAO representation of the nitrogen molecule, $\stackrel{33}{=}$ only the $2 \pi_{u}$ and the $2 \pi_{g}$ molecular orbitals (MOs) which are the two MOs whose occupancies change during the de-excitation process. To construct the two MOs we use moreover hydrogen-like wave functions with effective charges to mimic the RoothaanHartree-Fock wave functions of atomic nitrogen ${ }^{34}$ In the molecule's ground state $N_{2}\left({ }^{1} \Sigma_{g}^{+}\right)$the $2 \pi_{u}$ MO is fully occupied and the $2 \pi_{g}$ MO is empty while in the excited state $N_{2}\left({ }^{3} \Sigma_{u}^{+}\right)$the $2 \pi_{u}$ MO contains a hole and the $2 \pi_{g}$ $\mathrm{MO}$ is singly occupied. Both of these levels can carry four electrons and are degenerate in the electron spin $s= \pm \frac{1}{2}$ and the magnetic quantum number $m= \pm 1$. Since the process we consider does not involve any spin flip, we ignore the spin. We can thus label the ground state of the two-level system and its excited state by $0 \mathrm{~m}$ and $1 \mathrm{~m}$, respectively, and denote the corresponding energies by $\varepsilon_{0}$ and $\varepsilon_{1}$. The states of the metal and the free states are labelled by $\vec{k}$ and $\vec{q}$, respectively. The mathematical expressions for the wave functions of the single-electron states are given in Appendix A.

The description of the electronic structure of the molecule-surface system is completed by aligning the single-electron states against each other and against the vacuum level by use of the metal's work function $\Phi_{W}$, the metal's conduction band depth $\Phi_{C}$, the molecule's ionization energy $\Delta \varepsilon_{i}$, and the excitation energy of the molecule $\Delta \varepsilon_{e}$. The metal states are of course occupied up to the Fermi level $\varepsilon_{F}$. Our model is thus characterized by a few energy parameters, an effective charge, and a bond length (which enters the molecular wave functions of the diatomic molecule).

The electronic structure of the simplified model is sketched in Fig. 2, together with the transitions of the Penning de-excitation and its associated exchange process. Due to the symmetries of the molecular ground state $\left({ }^{1} \Sigma_{g}^{+}\right)$and the molecular excited state $\left({ }^{3} \Sigma_{u}^{+}\right)$, only transitions with $\Delta m=0$ are involved. They are driven by the Coulomb interaction between the excited electron in the $2 \pi_{g} \mathrm{MO}$ and an electron in the Fermi sea of the metal (see, for instance, Refs. 24 27). The three elec- 


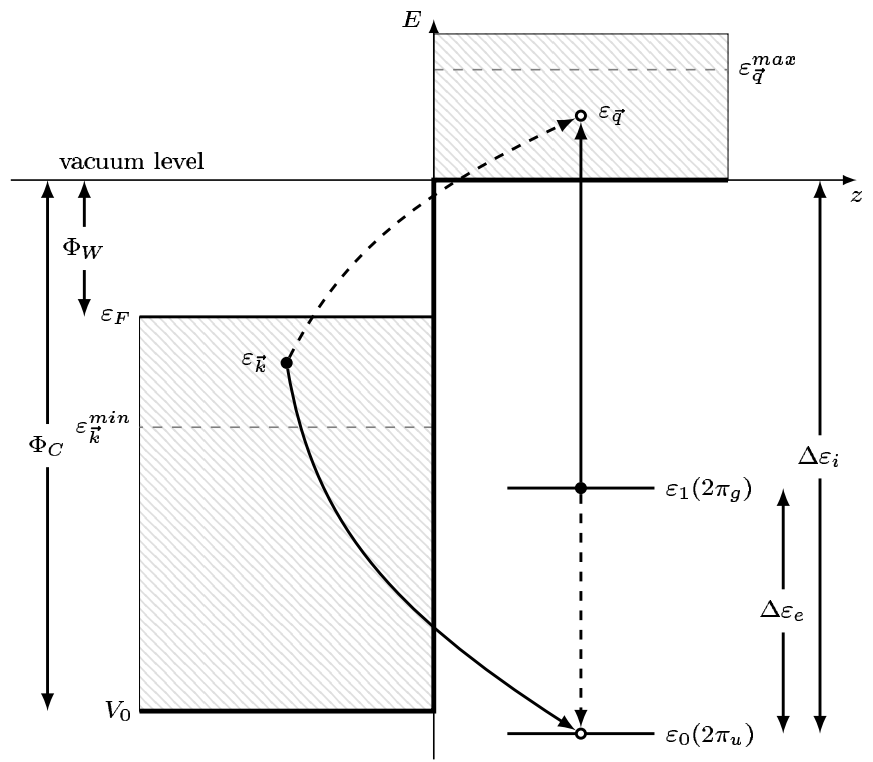

FIG. 2: Energy scheme of the simplified model showing Penning de-excitation (solid lines) and its exchange process (dashed lines). Also indicated are the classical energy cutoffs $\varepsilon_{\vec{k}}^{\min }$ and $\varepsilon_{\vec{q}}^{\max }$ which can be calculated from the energy balance $\varepsilon_{1}+\varepsilon_{\vec{k}}=\varepsilon_{0}+\varepsilon_{\vec{q}}$ that holds in the adiabatic limit.

trons in the $2 \pi_{u} \mathrm{MO}$ act only as spectators and can thus be neglected. Assuming moreover the Fermi surface of the metal to be rigid, the de-excitation of $N_{2}\left({ }^{3} \Sigma_{u}^{+}\right)$is basically a two-body scattering process, whose Hamiltonian, written in the single-electron basis described in the previous paragraph, is given by

$$
\begin{aligned}
H= & H_{0}+H_{1}(t), \\
H_{0}= & \sum_{\vec{k}} \varepsilon_{\vec{k}} c_{\vec{k}}^{\dagger} c_{\vec{k}}+\sum_{\vec{q}} \varepsilon_{\vec{q}} c_{\vec{q}}^{\dagger} c_{\vec{q}} \\
& +\sum_{m} \varepsilon_{0} c_{0 m}^{\dagger} c_{0 m}+\sum_{m} \varepsilon_{1} c_{1 m}^{\dagger} c_{1 m}, \\
H_{1}(t)= & \sum_{\vec{k}, \vec{q}, m} V_{0 m, \vec{k}}^{\vec{q}, 1 m}(t) c_{0 m}^{\dagger} c_{\vec{k}} c_{\vec{q}}^{\dagger} c_{1 m}+H . c .,
\end{aligned}
$$

where $H_{0}$ represents the Hamiltonian of the noninteracting system, and $H_{1}(t)$ contains the Penning deexcitation.

The Auger matrix element $V_{0 m, \vec{k}}^{\vec{q}, 1 m}$ contains the timedependence of the Hamiltonian, and thus carries the intrinsic non-equilibrium character of the system. In terms of the single-electron states given in Appendix A, it can be written as

$$
\begin{aligned}
V_{0 m, \vec{k}}^{\vec{q}, 1 m}(t)= & \int d \vec{r} \int d \vec{r}^{\prime} \Psi_{0 m}^{*}\left(\vec{r}_{\varphi}(t)\right) \Psi_{\vec{k}}(\vec{r}) \\
& \times V_{C}\left(\left|\vec{r}-\vec{r}^{\prime}\right|\right) \Psi_{\vec{q}_{\varphi}}^{*}\left(\vec{r}_{\varphi}^{\prime}(t)\right) \Psi_{1 m}\left(\vec{r}_{\varphi}^{\prime}(t)\right),
\end{aligned}
$$

where $V_{C}$ represents the Coulomb potential and the subscript $\varphi$ denotes the associated vector as seen from the molecule's reference frame, which is centered about the molecule's center of mass $\vec{R}(t)$ and has its $z$-axis aligned along the molecule axis. The vectors $\vec{r}_{\varphi}^{(\prime)}(t)$ and $\vec{q}_{\varphi}$ are thus given by

$$
\begin{aligned}
\vec{r}_{\varphi}^{(\prime)}(t) & =\hat{\Omega}(\varphi)\left(\vec{r}^{(\prime)}-\vec{R}(t)\right) \\
\vec{q}_{\varphi} & =\hat{\Omega}(\varphi) \vec{q}
\end{aligned}
$$

where the matrix $\hat{\Omega}(\varphi)$ describes the rotation around the $y$-axis (see Fig. 1). Due to the diatomicity of the nitrogen molecule the interaction matrix element depends on the orientation of the molecule with respect to the surface, that is, on the angle $\varphi$. For convenience we suppress however this dependence in our notation of the matrix element. Note, since we are considering the Penning process, the continuum states $\vec{q}$ are defined with respect to the molecule's center of mass. Thus the associated wave function within Eq. (7) must depend on the molecular reference frame variables $\vec{q}_{\varphi}$ and $\vec{r}_{\varphi}^{\prime}(t)$.

As already mentioned, we take the image interaction only for the emitted electron into account. It always feels the image potential and can thus only escape from the surface when its perpendicular energy is sufficiently high.$\underline{7}$ Therefore, in the calculation of the secondary electron emission coefficient and the spectrum of the Auger electron we will multiply the matrix element by a step function

$$
\Theta\left(\varepsilon_{q_{z}}-\frac{e^{2}}{16 \pi \varepsilon_{0}} \frac{1}{z_{R}(t)-z_{i}}\right)
$$

where $z_{i}$ is the position of the image plane. For aluminum and tungsten $z_{i}$ is given in Table $\square$ and turns out to be closer to the surface than the turning point of the molecule. Equation (9) is thus well-defined along the trajectory of the molecule. It is sometimes referred to as surface transmission function,,$\underline{7}$ although for the case of Penning de-excitation this term is somewhat misleading, as the escaping electron is not emitted from the inside of the solid but from the molecule.

\section{QUANTUM KINETICS}

The model established in the previous section will now be treated using the Keldysh technique, a brief description of which is given in Appendix B]

We start by calculating the unperturbed Green's functions $\mathcal{G}_{\alpha \beta}^{(0)}$, with $\alpha$ and $\beta$ representing any of the labels we used to characterize the single-electron states of the system, $\vec{k}, \vec{q}, 0 m$, or $1 m$. Since the time evolution of the free Green's functions is determined by $H_{0}$, they are diagonal, that is, $\mathcal{G}_{\alpha \beta}^{(0)} \sim \delta_{\alpha \beta}$. For convenience we abbreviate the double subscript $\alpha \alpha$ by $\alpha$ in the following. Inserting the solutions of the interaction-free Heisenberg equations for the creation and annihilation operators appearing in 
model (6), the free propagators read

$$
\begin{aligned}
i G_{\alpha}^{R(0)}\left(t, t^{\prime}\right) & =\Theta\left(t-t^{\prime}\right) e^{\frac{i}{\hbar} \varepsilon_{\alpha}\left(t-t^{\prime}\right)}, \\
i G_{\alpha}^{A(0)}\left(t, t^{\prime}\right) & =-\Theta\left(t^{\prime}-t\right) e^{\frac{i}{\hbar} \varepsilon_{\alpha}\left(t-t^{\prime}\right)}, \\
i G_{\alpha}^{K(0)}\left(t, t^{\prime}\right) & =\left[1-2 n_{\alpha}\left(t_{0}\right)\right] e^{\frac{i}{\hbar} \varepsilon_{\alpha}\left(t-t^{\prime}\right)},
\end{aligned}
$$

where $n_{\alpha}\left(t_{0}\right)$ is the initial occupancy of the state $\alpha$ at $t_{0}=-\infty$.

In accordance with the model we introduced in the previous paragraph we assume the excited molecular level to be initially occupied with a single electron of magnetic quantum number $m=\mu$, that is, $n_{1 m}\left(t_{0}\right)=\delta_{m \mu}$. The molecular ground state level, in contrast, is empty at $t_{0}$ because we neglect the spectator electrons. Hence, $n_{0 m}\left(t_{0}\right)=0$ for all $m$. The free electron states are also empty at $t_{0}$, implying $n_{\vec{q}}\left(t_{0}\right)=0$, and the electronic states within the metal are initially filled up to the Fermi energy $\varepsilon_{F}$, that is, $n_{\vec{k}}\left(t_{0}\right)=\Theta\left(\varepsilon_{F}-\varepsilon_{\vec{k}}\right)$.

For the calculation of the full Green's functions $\mathcal{G}_{\alpha \beta}$ we need expressions for the self-energies $\Sigma_{\alpha \beta}$, which, in line with the work by Makoshi,,$\frac{12}{2}$ we derive from a diagrammatic expansion up to second order in the Auger matrix element. This is justified because the Auger matrix element is in general very small. Because of the diagonality of the unperturbed Green's functions the self-energies and thus the full Green's function are also diagonal.

We first investigate the excited molecular state. Figure 3 shows the only non-vanishing second-order selfenergy diagram for $\Sigma_{1 \mu}$. It can be evaluated to

$$
\begin{aligned}
\Sigma_{1 \mu}^{R}\left(t_{1}, t_{2}\right)= & \Theta\left(t_{1}-t_{2}\right) \Sigma_{1 m}^{K}\left(t_{1}, t_{2}\right), \\
\Sigma_{1 \mu}^{A}\left(t_{1}, t_{2}\right)= & -\Theta\left(t_{2}-t_{1}\right) \Sigma_{1 m}^{K}\left(t_{1}, t_{2}\right), \\
\Sigma_{1 \mu}^{K}\left(t_{1}, t_{2}\right)= & -\frac{i}{\hbar^{2}} \sum_{\vec{q}, \vec{k}}\left[V_{0 \mu, \vec{q}}^{\vec{q}, 1 \mu}\left(t_{1}\right)\right]^{*} V_{0 \mu, \vec{k}}^{\vec{q}, 1 \mu}\left(t_{2}\right) \\
& \times n_{\vec{k}}\left(t_{0}\right) e^{-\frac{i}{\hbar}\left(\varepsilon_{0}+\varepsilon_{q}-\varepsilon_{k}\right)\left(t_{1}-t_{2}\right)} .
\end{aligned}
$$

Using Eq. (11a), the Dyson equation for the retarded Green's function (Eq. (B11a) in Appendix B) can be solved iteratively. The result is

$$
G_{1 \mu}^{R}\left(t, t^{\prime}\right)=G_{\mu}^{R(0)}\left(t, t^{\prime}\right) W_{\mu}\left(t, t^{\prime}\right)
$$

with the infinite series

$$
W_{\mu}\left(t, t^{\prime}\right)=\sum_{\nu=0}^{\infty} W_{\mu}^{(\nu)}\left(t, t^{\prime}\right)
$$

whose individual terms, $W_{\mu}^{(\nu)}$, are given by

$$
\begin{aligned}
W_{\mu}^{(\nu)}\left(t, t^{\prime}\right)= & (-1)^{\nu} \int_{t^{\prime}}^{t} d t_{1} \int_{t^{\prime}}^{t_{1}} d t_{2} \ldots \int_{t^{\prime}}^{t_{2 \nu-1}} d t_{2 \nu} \\
& \times \Delta_{\mu}\left(t_{1}, t_{2}\right) \ldots \Delta_{\mu}\left(t_{2 \nu-1}, t_{2 \nu}\right),
\end{aligned}
$$

where we introduced the quantity

$$
\Delta_{\mu}\left(t_{1}, t_{2}\right)=i \Sigma_{1 \mu}^{K}\left(t_{1}, t_{2}\right) e^{\frac{i}{\hbar} \varepsilon_{1}\left(t_{1}-t_{2}\right)},
$$

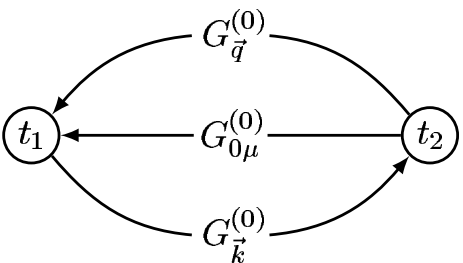

FIG. 3: Diagrammatic representation of the self-energy $\Sigma_{1 \mu}\left(t_{1}, t_{2}\right)$ of the excited molecular state in second order perturbation theory.

which emerges from the self-energy terms of the iterated Dyson equation.

The infinite series (13) is exact but useless. To obtain an expression for the retarded Green's function which is amenable to further manipulations we employ the exponential resummation technique (see, for instance, Ref. 28). For that purpose we introduce a new function $F_{\mu}\left(t, t^{\prime}\right)$ and perform a perturbation expansion of $W_{\mu}$ and $F_{\mu}$ in terms of $\Delta_{\mu}$. Using the virtual expansion parameter $\lambda=1$ we write

$$
\sum_{\nu=0}^{\infty} \lambda^{\nu} W_{\mu}^{(\nu)}\left(t, t^{\prime}\right)=e^{F_{\mu}\left(t, t^{\prime}\right)}=e^{\sum_{\nu=1}^{\infty} \lambda^{\nu} F_{\mu}^{(\nu)}\left(t, t^{\prime}\right)} .
$$

Expanding the exponential in (16) and then comparing the different orders of $\lambda$ leads to explicit expressions for the expansion coefficients $F_{\mu}^{(\nu)}$, the first few of which are

$$
\begin{aligned}
& F_{\mu}^{(1)}=W_{\mu}^{(1)}, \\
& F_{\mu}^{(2)}=W_{\mu}^{(2)}-\frac{1}{2}\left[W_{\mu}^{(1)}\right]^{2}, \\
& F_{\mu}^{(3)}=W_{\mu}^{(3)}-W_{\mu}^{(2)} W_{\mu}^{(1)}+\frac{1}{3}\left[W_{\mu}^{(1)}\right]^{3},
\end{aligned}
$$

where, for convenience, we dropped the time arguments.

The retarded Green's function can now be conveniently written as

$$
G_{1 \mu}^{R}\left(t, t^{\prime}\right)=G_{1 \mu}^{R(0)}\left(t, t^{\prime}\right) e^{F_{\mu}\left(t, t^{\prime}\right)}
$$

with the function $F_{\mu}$ in the exponent given by the sum of the terms in (17). Using relation (B10) together with Eq. (18) the advanced Green's function becomes

$$
G_{1 \mu}^{A}\left(t, t^{\prime}\right)=G_{1 \mu}^{A(0)}\left(t, t^{\prime}\right) e^{\left[F_{\mu}\left(t^{\prime}, t\right)\right]^{*}} .
$$

To calculate the occupation of the excited molecular state we also need the Keldysh part of the Green's function defined in Eq. (B12) of Appendix B. Using the explicit form of the free Green's functions (10) we first rewrite this equation into $\underline{12}$

$$
\begin{gathered}
G_{\alpha}^{K}\left(t, t^{\prime}\right)=-i\left[1-2 n_{\alpha}\left(t_{0}\right)\right] G_{\alpha}^{R}\left(t, t_{0}\right) G_{\alpha}^{A}\left(t_{0}, t^{\prime}\right) \\
\quad+\int_{t_{0}}^{t} d t_{1} \int_{t_{0}}^{t^{\prime}} d t_{2} G_{\alpha}^{R}\left(t, t_{1}\right) \Sigma_{\alpha}^{K}\left(t_{1}, t_{2}\right) G_{\alpha}^{A}\left(t_{2}, t^{\prime}\right)
\end{gathered}
$$


using the identity

$$
\begin{aligned}
G_{\alpha}^{K(0)}\left(t, t^{\prime}\right)= & -i\left[1-2 n_{\alpha}\left(t_{0}\right)\right] \\
& \times G_{\alpha}^{R(0)}\left(t, t_{0}\right) G_{\alpha}^{A(0)}\left(t_{0}, t^{\prime}\right),
\end{aligned}
$$

and the Dyson equation of the retarded and advanced Green's function (B11a). Note that Eq. (20) is not limited to the excited molecular level. It holds for all states $\alpha$.

Inserting (11c), (18), and (19) into (20) we can now calculate the Keldysh part of the Green's function $G_{1 \mu}^{K}\left(t, t^{\prime}\right)$. Taking the latter at equal times $t=t^{\prime}$ and utilizing Eq. (B13) we finally obtain for the occupancy of the excited level at time $t$,

$$
n_{1 \mu}(t)=e^{2 \Re\left[F_{\mu}\left(t, t_{0}\right)\right]},
$$

where $\Re[\ldots]$ denotes the real part. To lowest order in the interaction, that is, to lowest order in $\Delta_{\mu}$ the occupation of the molecular excited state is given by

$$
n_{1 \mu}^{(0)}(t)=e^{2 \Re\left[F_{\mu}^{(1)}\left(t, t_{0}\right)\right]}=e^{-\int_{t_{0}}^{t} d t_{1} \int_{t_{0}}^{t} d t_{2} \Delta_{\mu}\left(t_{1}, t_{2}\right)} .
$$

We now turn to the free electron states, that is, the states which may get occupied by the electron released by the de-excitation of the molecule. A treatment of these states analogous to the excited state leads to the following expression for the occupation at time $t$

$$
n_{\vec{q}}(t)=1-e^{2 \Re\left[F_{\mu, \vec{q}}\left(t, t_{0}\right)\right]},
$$

where $F_{\mu, \vec{q}}$ is defined the same way as $F_{\mu}$ but with $\Delta_{\mu}$ replaced by $\Delta_{\mu, \vec{q}}$. The latter is implicitly defined through the relation

$$
\Delta_{\mu}\left(t_{1}, t_{2}\right)=\sum_{\vec{q}} \Delta_{\mu, \vec{q}}\left(t_{1}, t_{2}\right)
$$

using Eqs. (15) and (11c) for $\Delta_{\mu}\left(t_{1}, t_{2}\right)$.

Equation (24) is not very useful, because it cannot easily be summed over $\vec{q}$, which is however needed to calculate the secondary electron emission coefficient $\gamma_{e}$. Because of this obstacle we adopt the approach of Makoshi ${ }^{12,13}$ and go back to Eq. (20), expand it for equal times $t=t^{\prime}$ and $\alpha=\vec{q}$ up to first order in the self-energies, and insert the result into Eq. (B13) which yields 13

$$
\begin{gathered}
n_{\vec{q}}(t)=-i \int_{t_{0}}^{t} d t_{1} \int_{t_{0}}^{t} d t_{2} G_{\vec{q}}^{R(0)}\left(t, t_{1}\right) \Sigma_{\vec{q}}^{K}\left(t_{1}, t_{2}\right) \\
\times G_{\vec{q}}^{A(0)}\left(t_{2}, t^{\prime}\right) .
\end{gathered}
$$

To account in Eq. (26) for lifetime effects of the metastable molecule we follow once more Makoshi ${ }^{12}$ and employ in the calculation of the self-energy $\Sigma_{\vec{q}}^{K}$ the full ("dressed") Green's function of the excited state $G_{1 \mu}^{+-}$instead of the unperturbed one. Up to second order (see

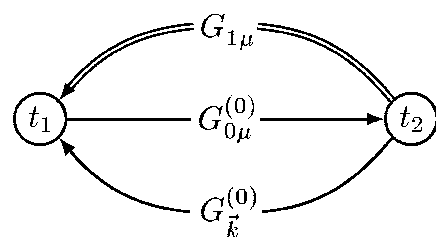

FIG. 4: Diagrammatic representation of the selfenergy $\Sigma_{\vec{q}}\left(t_{1}, t_{2}\right)$ of the emitted electron in second order perturbation theory. The dressed Green's function $G_{1 \mu}$ is indicated by a double line.

Fig. (4) we obtain

$$
\begin{aligned}
& \sum_{\vec{q}}^{K}\left(t_{1}, t_{2}\right)=\frac{1}{\hbar^{2}} \sum_{\vec{k}} V_{0 \mu, \vec{k}}^{\vec{q}, 1 \mu}\left(t_{1}\right)\left[V_{0 \mu, \vec{k}}^{\vec{q}, 1 \mu}\left(t_{2}\right)\right]^{*} \\
& \quad \times G_{0 \mu}^{-+(0)}\left(t_{2}, t_{1}\right) G_{\vec{k}}^{+-(0)}\left(t_{1}, t_{2}\right) G_{1 \mu}^{+-}\left(t_{1}, t_{2}\right) .
\end{aligned}
$$

In order to proceed we need to calculate $G_{1 \mu}^{+-}$. For that purpose, we first transform the corresponding component of the matrix Dyson equation (B7) into

$$
G_{1 \mu}^{+-}=G_{1 \mu}^{+-(0)}\left[1+\Sigma_{1 \mu}^{A} G_{1 \mu}^{A}\right]+G_{1 \mu}^{R(0)} \Sigma_{1 \mu}^{R} G_{1 \mu}^{+-}
$$

employing the fact that up to second perturbation order $\Sigma_{1 m}^{++} \equiv \Sigma_{1 \mu}^{R}$ and $\Sigma_{1 \mu}^{--} \equiv-\Sigma_{1 \mu}^{A}$. Inserting the free Green's functions (10) as well as the self-energies (11) and the full advanced Green's function (19) one can solve Eq. (28) iteratively to obtain

$$
G_{1 \mu}^{+-}\left(t, t^{\prime}\right)=G_{1 \mu}^{+-(0)}\left(t, t^{\prime}\right) e^{F_{\mu}\left(t, t_{0}\right)} e^{\left[F_{\mu}\left(t^{\prime}, t_{0}\right)\right]^{*}} .
$$

After inserting Eq. (10) and (29) into Eq. (27) we obtain for the Keldysh part of the self-energy

$$
\begin{aligned}
\Sigma_{\vec{q}}^{K}\left(t_{1}, t_{2}\right)= & \frac{i}{\hbar^{2}} \sum_{\vec{k}} \mathcal{V}_{0 \mu, \vec{k}}^{\vec{q}, 1 \mu}\left(t_{1}\right)\left[\mathcal{V}_{0 \mu, \vec{k}}^{\vec{q}, 1 \mu}\left(t_{2}\right)\right]^{*} \\
& \times n_{\vec{k}}\left(t_{0}\right) e^{\frac{i}{\hbar}\left(\varepsilon_{0}-\varepsilon_{k}-\varepsilon_{1}\right)\left(t_{1}-t_{2}\right)}
\end{aligned}
$$

with

$$
\mathcal{V}_{0 \mu, \vec{k}}^{\vec{q}, 1 \mu}(t)=V_{0 \mu, \vec{k}}^{\vec{q}, 1 \mu}(t) e^{F_{\mu}\left(t, t_{0}\right)}
$$

the renormalized Auger matrix element 12

To compute finally the occupation of the $\vec{q}$-states, we insert Eq. (30) into Eq. (26) and obtain

$$
n_{\vec{q}}(t)=\int_{t_{0}}^{t} d t_{1} \int_{t_{0}}^{t} d t_{2} \widetilde{\Delta}_{\mu, \vec{q}}\left(t_{1}, t_{2}\right)
$$

where $\widetilde{\Delta}_{\mu, \vec{q}}$ is defined in Eq. (25) with the plain matrix elements replaced by the renormalized matrix elements leading to

$$
\widetilde{\Delta}_{\mu, \vec{q}}\left(t_{1}, t_{2}\right)=\Delta_{\mu, \vec{q}}\left(t_{1}, t_{2}\right) e^{\left[F_{\mu}\left(t_{1}, t_{0}\right)\right]^{*}} e^{F_{\mu}\left(t_{2}, t_{0}\right)} .
$$


Equation (32) represents the spectrum of the emitted electrons. The secondary electron emission coefficient $\gamma_{e}$ can be calculated from Eq. (32) by taking $t=\infty$ and summing over all possible $\vec{q}$,

$$
\gamma_{e}=\sum_{\vec{q}} n_{\vec{q}}(\infty)=\int_{t_{0}}^{\infty} d t_{1} \int_{t_{0}}^{\infty} d t_{2} \widetilde{\Delta}_{\mu}\left(t_{1}, t_{2}\right)
$$

with $\widetilde{\Delta}_{\mu}$ defined by

$$
\widetilde{\Delta}_{\mu}\left(t_{1}, t_{2}\right)=\sum_{\vec{q}} \widetilde{\Delta}_{\mu, \vec{q}}\left(t_{1}, t_{2}\right) .
$$

Equation (34) can be rewritten to

$$
\gamma_{e}=1-e^{2 \Re\left[F_{\mu}\left(\infty, t_{0}\right)\right]} .
$$

The lowest two orders of Eq. (36) in terms $\Delta_{\mu}$ read

$$
\begin{aligned}
& \gamma_{e}^{(0)}=\int_{t_{0}}^{\infty} d t_{1} \int_{t_{0}}^{\infty} d t_{2} \Delta_{\mu}\left(t_{1}, t_{2}\right), \\
& \gamma_{e}^{(1)}=1-e^{-\int_{t_{0}}^{t} d t_{1} \int_{t_{0}}^{t} d t_{2} \Delta_{\mu}\left(t_{1}, t_{2}\right)} .
\end{aligned}
$$

The preceding calculation of the self-energies does not treat free and excited states on an equal footing. Only the self-energy for the free states is renormalized whereas the one for the excited state is not. As a result, particle conservation is not strictly guaranteed when the corresponding occupation numbers are calculated. The same shortcoming holds for Makoshi's ${ }^{12}$ original approach. Our numerical results showed however that particle conservation is obeyed for all physically relevant situations, justifying the treatment of the self-energies a posteriori.

Let us finally remark that although, as far as the logic of our approach is concerned, we closely followed Makoshi,, 12 our results have wider applicability. In contrast to him we do not work with a real phenomenological Auger interaction, depending only on time, and do not employ the wide-band approximation for the free states which would lead to time-local self-energies. We are also not restricted to the lowest order expressions given in Eq. (23) and (37). In principle, we can calculate corrections to these expressions using the higher order expansion coefficients of $F_{\mu}$ given by Eq. (17).

\section{NUMERICAL SCHEME}

Inspecting the main results of the previous section, Eq. (22) and (36), we realize that these equations are highly complex. For instance, calculating $\Delta_{\mu}$ from Eq. (15) requires summation of a matrix element product over all $\vec{k}$ and $\vec{q}$ vectors, which, letting the box size $L \rightarrow \infty$, equals a sixfold integral. The Auger matrix element itself, according to Eq. (7), involves another six dimensional integration over $\vec{r}$ and $\vec{r}^{\prime}$. Since in Eq. (15) the matrix element occurs as a product at two different times, this makes a total of eighteen dimensions of integration. Calculating the level occupancies from Eq. (22) and (36) requires at least another two-dimensional integration over the time arguments of $\Delta_{\mu}$. Thus, employing these equations as they stand for numerical calculations is clearly out of reach if reasonable computing time and considerably small numerical errors are required. In this section we will therefore introduce an approximation, which makes those numerical calculations feasible.

To simplify the matrix element (7) we first utilize the particular form of the wave functions (see Appendix A). Since the molecular wave functions $\Psi_{0 m / 1 m}$ are localized on the molecule and the metal wave functions $\Psi_{\vec{k}}$ and the free wave functions $\Psi_{\vec{q}}$ are bounded everywhere (in the mathematical sense), the main contribution to the matrix element (7) will arise from points close to the actual molecule position. Noting further, that for the low kinetic energies we are interested in $\left(\varepsilon_{\text {kin }} \leq 1 \mathrm{eV}\right)$ the molecule's turning point lies far outside the surface $\left(z_{0} \geq 4.35 a_{B}\right.$, see Eq. (4) and the parameters (5) $)$. We can thus safely restrict the $\vec{r}$-integration in (7) to $z \geq 0$, that is, neglect the overlap of the wave functions inside the solid. Using then the transformation

$$
\vec{r}^{(\prime)}=\Omega^{\dagger}(\varphi) \vec{r}_{1(2)}+\vec{R}(t)
$$

along with the explicit form of the electronic wave functions (see Appendix $\mathrm{A}$ ) we arrive at

$$
V_{0 m, \vec{k}}^{\vec{q}, 1 m}(t) \approx C_{V} T_{k_{z}} e^{-\kappa_{k_{z}} z_{R}(t)} V_{\vec{k} \vec{q}}^{m}
$$

with

$$
\begin{aligned}
C_{V}= & \frac{e^{2}}{4 \pi \varepsilon_{0}} \frac{\left[N_{\bar{Z}}^{q}\right]^{2}}{(2 \pi)^{\frac{5}{2}} \kappa^{2} \sqrt{N_{2 \pi_{u}} N_{2 \pi_{g}}}}, \\
V_{\vec{k} \vec{q}}^{m}= & \int d \vec{r}_{1} \int d \vec{r}_{2} \Theta\left(z_{1 \varphi}+z_{0}\right) \frac{\varrho_{1} \varrho_{2} e^{i m\left(\varphi_{2}-\varphi_{1}\right)}}{\left|\vec{r}_{1}-\vec{r}_{2}\right|}\left(e^{-\left|\vec{r}_{1+}\right|}+e^{-\left|\vec{r}_{1-}\right|}\right)\left(e^{-\left|\vec{r}_{2+}\right|}-e^{-\left|\vec{r}_{2-}\right|}\right) \\
& \times e^{i\left(k_{x} x_{1 \varphi}+k_{y} y_{1 \varphi}\right)} e^{-\kappa_{k_{z}} z_{1 \varphi}} e^{-i \vec{q}_{\varphi} \cdot \vec{r}_{2}} C\left(\vec{q}_{\varphi}, \vec{r}_{2+}, \bar{Z}\right) C\left(\vec{q}_{\varphi}, \vec{r}_{2-}, \bar{Z}\right)
\end{aligned}
$$




\begin{tabular}{c|r|r|r|r|r}
\hline \hline Material & $\Phi_{W}[e V]$ & $\Phi_{C}[e V]$ & Ref. & $z_{i}\left[a_{B}\right]$ & Ref. \\
\hline $\mathrm{Al}$ & 4.25 & 16.5 & $\mathbf{7}$ & 2.95 & $\underline{35}$ \\
$\mathrm{~W}$ & 4.5 & 10.9 & $\mathbf{1 8}$ & 3.0 & $\underline{35}$ \\
\hline \hline
\end{tabular}

TABLE I: Model parameters for aluminum and tungsten.

where $\varrho_{i}$ and $\varphi_{i}(i=1,2)$ are the usual cylindrical coordinates and we have introduced the abbreviations $\bar{Z}=Z_{C} / 2, \vec{r}_{i \pm}=\vec{r}_{i} \pm(\delta / 2) \vec{e}_{z}(i=1,2)$, and $\vec{r}_{1 \varphi}=$ $\left(x_{1 \varphi}, y_{1 \varphi}, z_{1 \varphi}\right)=\hat{\Omega}^{\dagger}(\varphi) \vec{r}_{1}$. Here $\delta$ is the bond length of the molecule. Note that in Eq. (40b) we have used $\kappa^{-1}$ as the unit of length. Moreover, we did approximate $\Theta\left(z_{1 \varphi}+z_{R}(t)\right)$ by $\Theta\left(z_{1 \varphi}+z_{0}\right)$, which, as numerical tests confirmed, is a good approximation for the distant turn- ing point positions we are considering.

To calculate the occupation numbers from the equations given in Sec. III we need to compute the time integral of $\Delta_{\mu}\left(t_{1}, t_{2}\right)$. Since in Eq. (39) the time dependence has been separated we can insert the trajectory (3) and carry out the time integration analytically. For the lowest order equations it suffices to calculate the integral with equal upper boundaries, that is,

$$
\mathcal{I}(t)=\int_{t_{0}}^{t} d t_{1} \int_{t_{0}}^{t} d t_{2} \Delta_{\mu}\left(t_{1}, t_{2}\right)
$$

Using spherical coordinates for the wave vectors $\vec{k}$ and $\vec{q}$ we obtain

$$
\begin{aligned}
\mathcal{I}(t)= & \frac{\bar{Z}^{2}}{2(2 \pi)^{8} N_{2 \pi_{u}} N_{2 \pi_{g}}} \frac{e^{4}}{\hbar^{2} v^{2} k_{V}^{2} \varepsilon_{0}^{2}} \int_{0}^{k_{F}} d k \int_{0}^{\pi} d \vartheta_{k} \int_{0}^{2 \pi} d \varphi_{k} \int_{0}^{q_{c}} d q \int_{0}^{\frac{\pi}{2}} d \vartheta_{q} \int_{0}^{2 \pi} d \varphi_{q} \\
& \times \frac{k^{4} \sin \left(\vartheta_{k}\right) \cos ^{2}\left(\vartheta_{k}\right) \sin \left(\vartheta_{q}\right)}{\left(1-e^{-2 \pi \frac{\bar{Z}}{q}}\right)^{2}} e^{-2 \kappa_{k_{z}} z_{0}}\left|V_{\vec{k} \vec{q}}^{\mu}\right|^{2}\left|\Gamma_{\vec{k} \vec{q}}(t)\right|^{2}
\end{aligned}
$$

where

$$
k_{F}=\sqrt{\frac{2 m_{e}\left(\varepsilon_{F}-V_{0}\right)}{\hbar^{2}}}, \quad k_{V}=\sqrt{\frac{2 m_{e}\left|V_{0}\right|}{\hbar^{2}}},
$$

and

$$
\begin{aligned}
\Gamma_{\vec{k} \vec{q}}(t)= & \Theta(-t) \frac{e^{\left(\kappa_{k_{z}}-i \Delta \varepsilon_{k q}\right) \min \left(t,-t_{\vec{q}}\right)}}{\kappa_{k_{z}}-i \Delta \varepsilon_{k q}}+\Theta(t)\left(\frac{e^{\left(\kappa_{k_{z}}-i \Delta \varepsilon_{k q}\right) \min \left(0,-t_{\vec{q}}\right)}}{\kappa_{k_{z}}-i \Delta \varepsilon_{k q}}\right. \\
& +\Theta\left(t-t_{\vec{q})} \frac{e^{-\left(\kappa_{k_{z}}+i \Delta \varepsilon_{k q}\right) \max \left(0, t_{\vec{q}}\right)}-e^{-\left(\kappa_{k_{z}}+i \Delta \varepsilon_{k q}\right) t}}{\kappa_{k_{z}}+i \Delta \varepsilon_{k q}}\right),
\end{aligned}
$$

with

$$
\begin{aligned}
\Delta \varepsilon_{k q} & =\frac{\varepsilon_{0}+\varepsilon_{q}-\varepsilon_{1}-\varepsilon_{k}}{\hbar \kappa v} \\
t_{\vec{q}} & =z_{i}-z_{0}+\frac{m_{e} e^{2}}{8 \pi \varepsilon_{0} \hbar^{2} \kappa} \frac{1}{q^{2} \cos ^{2}\left(\vartheta_{q}\right)} .
\end{aligned}
$$

The term $t_{\vec{q}}$ emerges from the inclusion of the transfer function (9) and needs to be set to zero when considering the population of the excited molecular level.

Equation (42) is still highly complex, as it consists of an integral over 12 variables. In order to compute this expression numerically we divide the calculation into two steps. First, we calculate $V_{\vec{k} \vec{q}}^{\mu}$ on a discrete grid within the $(\vec{k}, \vec{q})$-space. Afterwards, we calculate the wave vector integral in Eq. (42) while using sexa-linear interpolation to obtain the intergrid-values of $V_{\vec{k} \vec{q}}^{\mu}$. Because of this gridbased scheme we have to introduce a cut-off constant $q_{c}$ for the $q$-integration in Eq. (42), which we choose to be slightly larger than the classical cut-off $q_{\max }$. This is however not an issue, since the spectrum of the emitted electron falls off very rapidly beyond the classical cutoff. The high-dimensional integrals which occur in our formalism are then efficiently computable by means of Monte Carlo techniques. In particular, we employed the VEGAS algorithm as implemented in the GNU Scientific Library.

\section{RESULTS}

We now present numerical results. The parameter values used in our calculations are listed in Table I and III In all of the calculations we fixed the turning point $z_{0}$ to the value given by Eq. (4) for $50 \mathrm{meV}$, which 


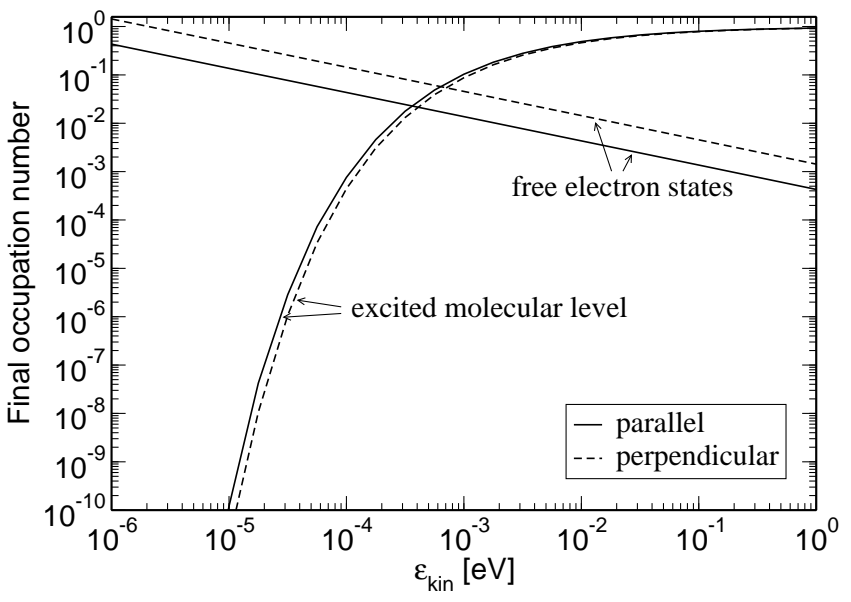

FIG. 5: Final occupancies of the excited molecular level and of the free electron states (secondary electron emission coefficient) in parallel (solid lines) and perpendicular (dashed lines) molecule orientation for different kinetic energies $\varepsilon_{k i n}$ of the incident molecule. The curves were calculated using the zeroth order formulas, Eq. (23) and (37a), respectively.

amounts to approximately $4.42 a_{B}$. Within the energy range $\varepsilon_{k i n} \leq 1 \mathrm{eV}$ this approximation is uncritical, since the turning point as given by Eq. (4) varies very weakly. We aim at calculating, respectively, the occupancies of the excited molecular state $n_{1}(t)$ and of the free electron states $n(t)=\sum_{\vec{q}} n_{\vec{q}}(t)$. Within our model the initial magnetic quantum number $\mu= \pm 1$ has no impact on the occupation numbers. For convenience we thus omit any $m$ subscripts in the following. We restrict the molecule's orientation to the two fundamentally distinct situations $\varphi=0$ (axis parallel to the surface) and $\varphi=\frac{\pi}{2}$ (axis perpendicular to the surface). Furthermore, if not stated otherwise, we consider an aluminum surface.

We start our analysis with the final occupancies, that is, $n_{1}(\infty)$ and $n(\infty)$ where, according to Eq. (34), the latter quantity is the secondary electron emission coefficient $\gamma_{e}$. For now we employ only the lowest order equations (23) and (37a), which, concerning the emitted electron, means that we neglect the matrix element renormalization and thus the lifetime effects of the metastable molecule.

The final occupancies of the excited molecular level and of the free electron states (secondary electron emission coefficient $\gamma_{e}$ ) are plotted in Fig. 5 for different kinetic energies of the incident molecule. Obviously, the Penning process gets more efficient for lower kinetic ener-

\begin{tabular}{c|r|r}
\hline \hline Parameter & Value & Reference \\
\hline$\delta$ & $2.067 a_{B}$ & $\underline{33}$ \\
$\varepsilon_{0}$ & $-17.25 \mathrm{eV}$ & $\underline{8}$ \\
$\varepsilon_{1}$ & $-9.57 \mathrm{eV}$ & $\underline{8}$ \\
\hline \hline
\end{tabular}

TABLE II: Model parameters for nitrogen.

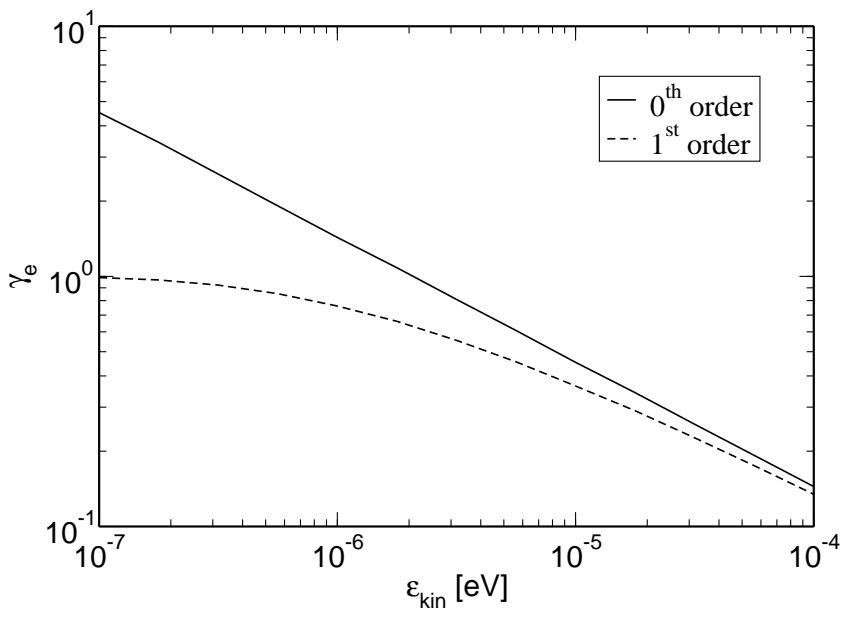

FIG. 6: Comparison of the secondary electron emission coefficient $\gamma_{e}$ computed in zeroth order from Eq. (37a) (solid line) and in first order from Eq. (37b) (dashed line). The molecular axis was aligned perpendicular to the surface. Clearly, the first order correction heals the divergence of $\gamma_{e}$ for $\varepsilon_{k i n} \rightarrow 0$ and ensures $\gamma_{e} \leq 1$ for all energies.

gies, which is evident, because lower kinetic energies correspond to smaller molecule velocities and thus to larger interaction times of the solid-molecule system. Note, that for $\varepsilon_{k i n} \leq 10^{-6} \mathrm{eV}$ the secondary electron emission coefficient gets larger than one although physical values for $\gamma_{e}$ should obviously be less than or equal to one. This unphysical peculiarity is a consequence of the negligence of the lifetime effect in the zeroth order formula for $\gamma_{e}$, Eq. (37a), and can be fixed by employing higher order terms of the full Eq. (36) (see below).

From Fig. 5 we see that for the two distinct orientations the Penning process is almost equally efficient in deexciting the molecule. The number of emitted electrons, however, is about a factor three smaller when the molecular axis is parallel to the surface as compared to when it is perpendicular. Numerical tests showed that for the parallel case the electron is primarily emitted with a very small perpendicular energy whereas in the perpendicular case the perpendicular energy of the emitted electron is distributed more equally. Thus, in the perpendicular orientation the electron has a higher probability to breach through the surface barrier originating from the image potential leading to a larger value for $\gamma_{e}$.

To fix the unphysical behavior of the secondary electron emission coefficient at low collision energies, the first order formula, Eq. (37b), already suffices. It includes additional exponential factors which damp the integrand in the divergent region leading to $\gamma_{e} \rightarrow 1$ for $\varepsilon_{k i n} \rightarrow 0$. Figure 6 explicitly demonstrates this behavior. The region in which the zeroth order result for $\gamma_{e}$ exceeds unity corresponds to very low molecule velocities $\varepsilon_{k i n} \leq 10^{-6} \mathrm{eV}$. These sub-thermal collision energies are rarely realized and are of lesser significance to our problem. In lowtemperature plasmas, for instance, the systems we are primarily interested in, molecules have at least thermal 

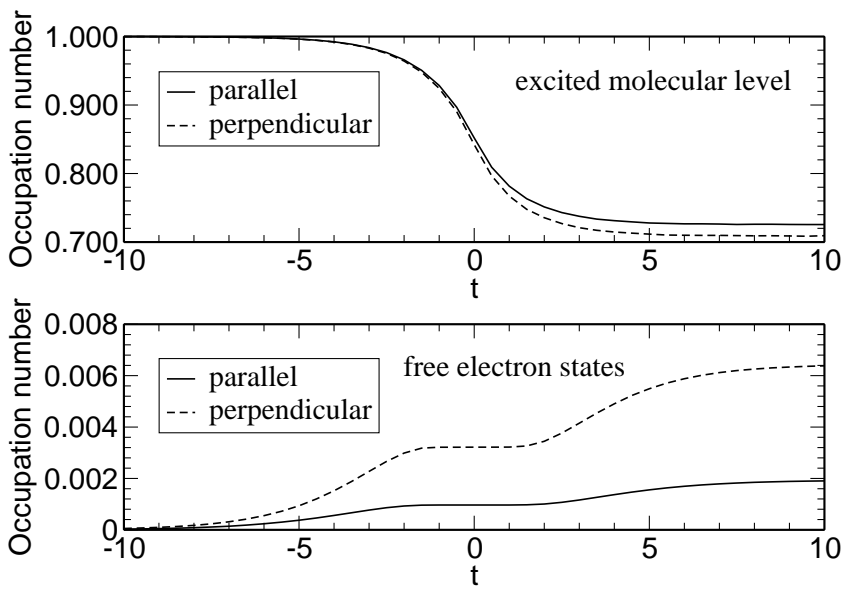

FIG. 7: Time evolution of the occupancies of the excited molecular level (upper panel) and of the free electron states (lower panel) for parallel (solid lines) and perpendicular (dashed lines) molecule orientation. The kinetic energy of the incident molecule was fixed to $50 \mathrm{meV}$. Time is measured in units of $1 /(\kappa v)$. A time difference of $\Delta t=1$ thus corresponds to the motion of the molecule over a distance of $\Delta z=a_{B} / 2$.

collision energies. Only in beam experiments with extreme grazing incidence ${ }^{4}$ may the collision energies be low enough to require the lifetime effect to be explicitly included in a theoretical analysis. For electron energies above $0.1 \mathrm{meV}$ the difference between the zeroth and first order formulas are vanishingly small indicating that in this energy range Eq. (37a) is sufficient.

Next, we investigate the time evolution of the occupancies. We fix the kinetic energy of the molecule to $50 \mathrm{meV}$, which is about twice the thermal energy at room temperature. In addition, we employ the zeroth order formulas. This is justified because the higher order corrections are small in the considered energy region. The results are plotted in Fig. 7 Obviously, the occupancy of the excited molecular level (upper panel) changes significantly only in the range $|t| \leq 5$, which, taking the turning point into account, equals maximum distances of the molecule's center of mass from the surface of roughly $7 a_{B}$. As expected, within our model, the process is equally effective in the incoming and outgoing branch of the trajectory. The time evolution of the occupancy of the free states (lower panel) is however distinctively different. It shows a plateau around $t=0$, that is, a stagnation of the probability to escape from the solid. This is a consequence of the image potential which almost completely traps an electron emitted at low surface distances where its perpendicular energy is too small to overcome the image barrier encoded in the surface transmission function.

The energy distribution $n_{\varepsilon_{\vec{q}}}$ of the emitted electron at $t=\infty$ is also of interest. This quantity is shown in Fig. 8 for the two principal molecule orientations and a collision energy $\varepsilon_{k i n}=50 \mathrm{meV}$. The graphs for the two different orientations (denoted by "this work") start at the origin and monotonously increase until a cut-off energy is

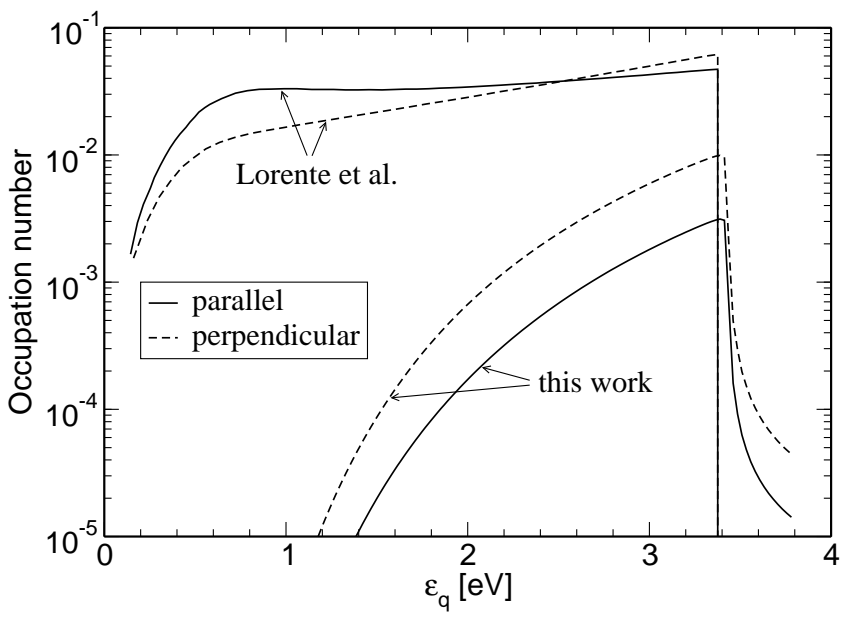

FIG. 8: Energy spectrum $n_{\varepsilon_{\vec{q}}}(\infty)=n_{\vec{q}}(\infty)$ of the Penningemitted electron for parallel and perpendicular molecule orientation (reaction (1), this work). The kinetic energy of the incident molecule was fixed to $50 \mathrm{meV}$. For comparison we also show the results Lorente et al..$^{7}$ obtained for reaction (2).

reached. The latter resembles the classical cut-off energy $\varepsilon_{\vec{q}}^{\max }$ (see Fig. 2), implying that energy conservation is restored at the end of the collision, as it should be. The low energy part of the spectrum is cut off due to the surface transmission function which allows electrons to escape from the surface only when their perpendicular energy is large enough. The spectrum for the perpendicular case takes on larger values, as was explained in connection with the $\vec{q}$-integrated spectrum shown in Fig. 5 .

For comparison, we also plotted in Fig. 8 the spectra Lorente et al. 7 obtained for an electron released due to charge-transfer and subsequent auto-detachment, reaction (2), assuming $z_{0} \approx 5 a_{B}$. For low energies the spectra due to Penning de-excitation (1) are practically zero and thus significantly smaller than the ones corresponding to reaction (2). In this energy range the resonant channel dominates. Close to the cut-off energy, however, the Auger spectra are only one order of magnitude smaller than the spectra for the charge-transfer process indicating that in this energy range the two processes are indeed eye-to-eye competitors. To study in our model the competition between the two channels quantitatively requires however to dress-up the free Green's functions of the molecular electron by a hybridization self-energy and is thus beyond the scope of the present investigation. Integration of the energy spectra yields the secondary electron emission coefficients $\gamma_{e}$ listed in Table III. For aluminum the Penning process is thus one to two orders of magnitude weaker than the resonant process.

We now investigate the influence of the molecule's turning point on the de-excitation process. For aluminum and tungsten the occupation number of the excited molecular level calculated with the turning point given by Eq. (4) for $\varepsilon_{k i n}=50 \mathrm{meV}$ is shown in Fig. 9 together with the data obtained for the turning point 


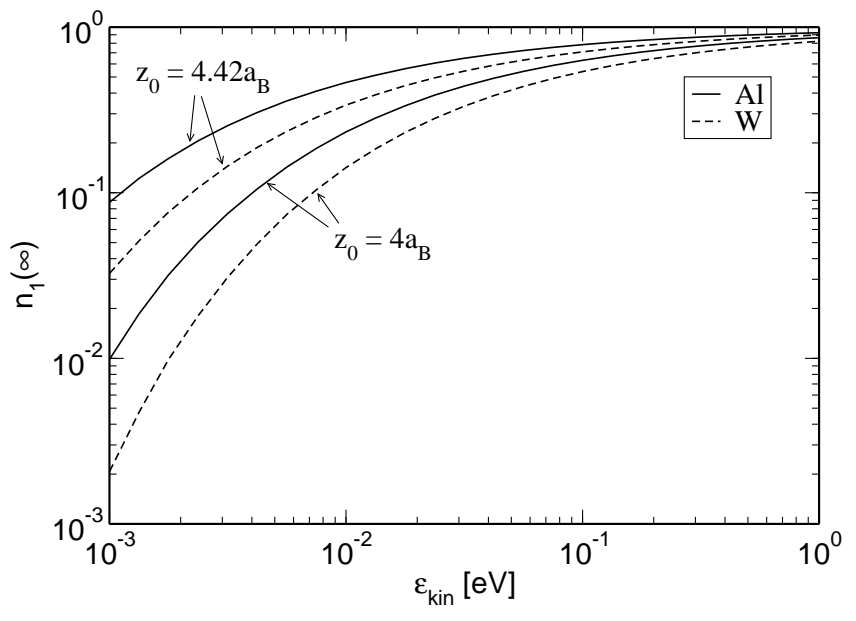

FIG. 9: Comparison of the final occupancy of the excited molecular level $n_{1}(\infty)$ for two different values of the turning point $z_{0}$ for aluminum (solid lines) and tungsten (dashed lines). The molecule's axis is perpendicular to the surface.

fixed to $z_{0}=4 a_{B}$. For the latter the de-excitation probability increases drastically at low energies leading to significantly reduced occupancies of the excited molecular state. At large energies the difference in the turning points shows almost no influence. Quantitatively, for energies below $1 \mathrm{meV}$, the occupancy of the excited molecular level for $z_{0}=4 a_{B}$ is approximately one order of magnitude smaller as the one obtained for $z_{0}=4.42 a_{B}$. This has however no influence for the occupancy of the free states (not shown in Fig. 9) because an electron emitted closer to the surface has a lower perpendicular energy and can thus no longer breach through the surface potential arising from the image potential as we discussed before.

Throughout our investigation we assumed that the Coulomb interaction, which drives the Penning deexcitation of the molecule, is unscreened. In reality, however, the Coulomb interaction in the vicinity of a surface is screened due to the charge carriers of the solid. The strength of Penning de-excitation should thus be affected by screening. To estimate this effect it suffices to consider the statically screened Coulomb potential $V_{C}^{S}(r)=V_{C}(r) e^{-\kappa_{s} r}$ with $\kappa_{s}$ the screening wave number at the surface. Little is known about this quantity

\begin{tabular}{c|r|r}
\hline \hline & $\gamma_{e}$ (parallel) & $\gamma_{e}$ (perpendicular) \\
\hline This work & $1.93 \cdot 10^{-3}$ & $6.43 \cdot 10^{-3}$ \\
Lorente et al. & $1.06 \cdot 10^{-1}$ & $8.98 \cdot 10^{-2}$ \\
\hline \hline
\end{tabular}

TABLE III: Comparison of the secondary electron emission coefficient $\gamma_{e}$ due to Penning de-excitation (this work) and resonant charge-transfer with subsequent auto-detachment (Lorente et al. ${ }^{7}$ ) for a $N_{2}\left({ }^{3} \Sigma_{u}^{+}\right)$molecule hitting an aluminum surface with a kinetic energy of $50 \mathrm{meV}$ and parallel or perpendicular orientation. except that it has to be smaller then the bulk screening wave number. Positron transmission and trapping experiments for various metallic films ${ }^{36}$ indicated, for instance, that the screening wave number near the surface is most probably a factor 0.6 less than in the bulk. Taking this correction factor into account the screening wave number for an aluminum surface, for instance, is $1.2285 / \AA$. The screened Coulomb potential affects only the values of $V_{\vec{k} \vec{q}}^{\mu}$, which enters quadratically into the lowest order formulas for the occupation numbers and reduces them by approximately $40 \%$. Thus, as expected, screening reduces the efficiency of the Coulomb-driven de-excitation channel, but it does not change its order of magnitude.

So far, we compared our results only with the theoretical results of Lorente et al. $\frac{7}{7}$ who studied the chargetransfer channel (2) for $N_{2}\left({ }^{3} \Sigma_{u}^{+}\right)$hitting an aluminum surface. To make contact to the experimental data obtained by Stracke et al. 6 , who also emphasized the direct charge-transfer reaction but nevertheless gave an estimate for $\gamma_{e}$ due to Penning de-excitation (1), we also calculated $\gamma_{e}$ for tungsten using the $50 \mathrm{meV}$ turning point of the surface potential (44) and a statically screened Coulomb interaction with $\kappa_{s}=0.6 \kappa_{b}^{W}$ where $\kappa_{b}^{W}=1.7659 / \AA$ is the screening wave number of bulk tungsten $\stackrel{37}{=}$ We find $\gamma_{e} \approx 2 \cdot 10^{-3}$ which is rather close to the experimental estimate $\left.\gamma_{e}^{\exp }\right|_{\text {Penning }} \approx 10^{-4}-10^{-3}$. Despite the crudeness of the effective model, which neglects, for instance, dangling bonds and surface states and the simplistic treatment of screening near a surface, our approach seems to capture even quantitatively the essential physics of Penning de-excitation. We attribute this to the rather large value of the molecule's turning point $z_{0} \approx 4.42 a_{B}$ which partly immunizes Penning deexcitation against the details of the electronic structure in the immediate vicinity of the surface.

\section{CONCLUSIONS}

We investigated the release of secondary electrons due to Auger de-excitation of metastable nitrogen molecules at metallic surfaces using an effective model for the two active electrons involved in the process and the Keldysh formalism to calculate the occupation numbers of the relevant single-electron states as originally proposed by Makoshi.12 In contrast to him, however, we are not restricted to time-local Auger self-energies and thus to the wide-band approximation because we solve the Dyson equation for the retarded Green's function by exponential resummation. We also employed the Auger matrix element obtained from a LCAO-type description of the molecule and an abrupt half-space-type description of the metal and not a phenomenological matrix element. The dependencies of the matrix element on the single-electron quantum numbers are retained in our calculation, as is the image interaction of the Auger electron with the surface and the distortion of the continuum of Auger states in the vicinity of the surface due to the molecule which 
is accounted for by using for the Auger electron a continuum of two-center Coulomb waves.

The complexity of the final equations forced us to calculate and interpolate the Auger matrix element on a grid in a high-dimensional parameter space. This numerical approximation for the matrix element enabled us however to calculate the time evolution and the final values of the occupancies of the excited molecular level and of the free electron states with standard Monte-Carlo integration routines without further approximations. Since the Auger interaction is rather weak, we utilized only the lowest order formulas derived from the quantum kinetic theory. The lifetime correction introduced by Makoshi,,$\frac{12}{,}$ contained in higher order terms, was shown to be important only for very low kinetic energies of the molecule.

We applied our approach to Auger de-excitation of $N_{2}\left({ }^{3} \Sigma_{u}^{+}\right)$on aluminum or tungsten. For an aluminum surface we showed that for realistic turning points of the molecule's trajectory, for instance, the one obtained from the molecular-surface potential for thermal kinetic energies, which is about $4.42 a_{B}$, Auger de-excitation is one to two orders of magnitude less efficient in releasing an electron than the direct charge-transfer process investigated by Lorente and coworkers $\stackrel{\underline{7}}{\underline{y}}$ For a tungsten surface our model produced for a turning point of $4.42 a_{B}$ a secondary electron emission coefficient due to Auger de-excitation which agrees with the experimental estimates of Stracke and coworkers $\underline{\underline{6}}$

The effective model we used works with crude wave functions, but has the virtue to be parameterizable with a few easily obtainable energies. For the applications we have in mind, secondary electron emission in lowtemperature gas discharges, where a great variety of different kinds of molecules and different kinds of wall materials occur, we consider this as a real advantage. With appropriate modifications the model can be applied to dielectric surfaces as well. We used the model only for the investigation of Auger de-excitation. In combination with the Keldysh Green's function technique, however, it can be also employed for the description of direct charge-transfer processes provided the energy shift and the broadening of the molecular levels due to the image interaction of the molecule with the surface and the self-energy corrections due to the Coulomb interaction between the excess electron and the excited electron of the molecule are included.

\section{Acknowledgments}

Johannes Marbach was funded by the International Max Planck Research School on Bounded Plasmas. In addition this work was supported by the Deutsche Forschungsgemeinschaft through the Transregional Collaborative Research Center SFB/TRR24.

\section{Appendix A: Wave functions}

The bound wave functions for the active molecular electron $\Psi_{0 m / 1 m}$ are calculated from the linear combination of atomic orbitals

$$
\Psi_{0 m / 1 m}=\Psi_{2 \pi_{u / g}^{m}}=\frac{\Psi_{2 p_{m}}(1) \pm \Psi_{2 p_{m}}(2)}{N_{2 \pi_{u / g}}}
$$

with 1 and 2 labeling the two distinct nitrogen atoms and $N_{2 \pi_{u / g}}$ denoting normalization constants. The atomic nitrogen wave functions $\Psi_{2 p_{m}}$ are approximated by using a hydrogen-like model with effective nucleus charge $Z_{\text {eff }}=4$. The resulting wave functions show excellent agreement with Roothaan-Hartree-Fock calculations for the nitrogen atom $\stackrel{34}{*}$ The molecular wave functions (A1) are most conveniently expressed in cylindrical coordinates $(\varrho, \varphi, z)$ and possess the explicit form

$$
\begin{aligned}
& \Psi_{2 \pi_{u / g}^{m}}(\varrho, \varphi, z)=\frac{1}{N_{2 \pi_{u / g}}} \frac{-2 m \kappa^{\frac{5}{2}}}{\sqrt{8 \pi}} \varrho e^{i m \varphi} \\
& \times\left(e^{-\kappa \sqrt{\varrho^{2}+\left(z+\frac{\delta}{2}\right)^{2}}} \pm e^{-\kappa \sqrt{\varrho^{2}+\left(z-\frac{\delta}{2}\right)^{2}}}\right),
\end{aligned}
$$

with $\kappa=2 / a_{B}$ and normalization constants

$$
\begin{aligned}
N_{2 \pi_{u / g}}= & 2 \pm 2 \kappa^{5} \int_{0}^{\infty} d \varrho \int_{-\infty}^{\infty} d z \varrho^{3} \\
& \times e^{-\kappa \sqrt{\varrho^{2}+\left(z+\frac{\delta}{2}\right)^{2}}} e^{-\kappa \sqrt{\varrho^{2}+\left(z-\frac{\delta}{2}\right)^{2}}}
\end{aligned}
$$

which need to be calculated numerically. The order of the wave functions within (A1), that is, the labeling of the nitrogen atoms does not matter in our calculation.

The wave functions for the electrons inside the metal are calculated along the lines of Ref. 25 by solving the Schrödinger equation for an electron trapped by the step potential

$$
V(z)=\left\{\begin{array}{ll}
-\left|V_{0}\right| & z<0 \\
0 & z \geq 0
\end{array} .\right.
$$

The presence of the molecule is thus ignored, as far as the calculation of the metal wave functions is concerned. Using box normalization with box size $L$ we obtain

$$
\begin{aligned}
\Psi_{\vec{k}}(\vec{r})= & \frac{1}{L \sqrt{L}} e^{i\left(k_{x} x+k_{y} y\right)}\left\{T_{k_{z}} e^{-\kappa_{k_{z}} z} \Theta(z)\right. \\
& \left.+\left[e^{i k_{z} z}+R_{k_{z}} e^{-i k_{z} z}\right] \Theta(-z)\right\},
\end{aligned}
$$

where the following wave vector dependent coefficients have been introduced

$$
\begin{aligned}
R_{k_{z}} & =\frac{i k_{z}+\kappa_{k_{z}}}{i k_{z}-\kappa_{k_{z}}}, \\
T_{k_{z}} & =\frac{2 i k_{z}}{i k_{z}-\kappa_{k_{z}}}, \\
\kappa_{k_{z}} & =\sqrt{\frac{2 m_{e}}{\hbar^{2}}\left|V_{0}\right|-k_{z}^{2}} .
\end{aligned}
$$


The energy of an electron in the state $\vec{k}$ is given by

$$
\varepsilon_{\vec{k}}=\frac{\hbar^{2}}{2 m_{e}}\left(k_{x}^{2}+k_{y}^{2}+k_{z}^{2}\right)-\left|V_{0}\right| .
$$

We now turn to the free states involved in the Penning process. As discussed in the main text we include here the effect the molecule has because the Auger electron originates from the molecule. The continuum of free states resembles thus not the continuum of the solid but the single-electron continuum of the molecule which we approximate by a two-center Coulomb (TCC) wave $\underline{\underline{30}}$ In the past it has been successfully used to model electronimpact ionization and photo-ionization of $\mathrm{H}_{2}$ and $\mathrm{H}_{2}^{+}$ molecules ${ }^{31.32}$ The TCC wave is an approximate solution of Schrödinger's equation for an unbound electron moving in the field of two fixed centers. Employing $a_{B}$ as the unit of length it reads

$$
\Psi_{\vec{q}}(\vec{r})=\frac{e^{i \vec{q} \cdot \vec{r}}}{(2 \pi)^{\frac{3}{2}}} N_{Z_{1}}^{q} C\left(\vec{q}, \overrightarrow{r_{1}}, Z_{1}\right) N_{Z_{2}}^{q} C\left(\vec{q}, \overrightarrow{r_{2}}, Z_{2}\right)
$$

with

$$
\begin{gathered}
N_{Z}^{q}=e^{\frac{\pi}{2} \frac{Z}{q}} \Gamma\left(1+i \frac{Z}{q}\right), \\
C(\vec{q}, \vec{r}, Z)=M\left(-i \frac{Z}{q}, 1,-i[q r+\vec{q} \cdot \vec{r}]\right),
\end{gathered}
$$

where $M$ is the confluent hypergeometric function of the first kind. The two vectors $\vec{r}_{1}$ and $\vec{r}_{2}$ denote, respectively, the position of the electron as seen from nucleus 1 and 2 and $Z_{1}$ and $Z_{2}$ are effective charge numbers. As proposed in Ref. 31 we account for the partial screening of the nuclei by the passive electrons and choose $Z_{1}=Z_{2}=Z_{C}=\frac{1}{2}$.

\section{Appendix B: Keldysh formalism}

To fix our notation we give a brief description of the Keldysh formalism. For a more complete survey of the topic we refer the reader to Refs. $14-17$.

Consider a fermionic system with a Hamiltonian

$$
H(t)=H_{0}+H_{1}(t)
$$

where $H_{1}(t)$ represents a time-dependent perturbation of the non-interacting system $H_{0}$. Due to the timedependence of the Hamiltonian $H(t)$ we are faced with a non-equilibrium situation.

One way to treat systems with a time-dependent Hamiltonian is the non-equilibrium Green's function technique introduced by Keldysh $\underline{\underline{14}}$ The key feature of the technique is a time contour $\mathcal{C}$ in the complex plane running from $-\infty$ to $\infty$ and then back again to $-\infty$. All quantities of the usual Green's function technique are then defined on this complex time path.
Of particular importance is the contour-ordered Green's function $\mathcal{G}_{\alpha \beta}\left(t, t^{\prime}\right)$, describing the propagation from a state $\beta$ at time $t^{\prime}$ to a state $\alpha$ at time $t$. It is defined by

$$
i \mathcal{G}_{\alpha \beta}\left(t, t^{\prime}\right)=\left\langle T_{\mathcal{C}}\left[\Psi_{\alpha}(t) \Psi_{\beta}^{\dagger}\left(t^{\prime}\right)\right]\right\rangle_{H},
$$

where $T_{\mathcal{C}}$ specifies the chronological time-ordering operator on the contour, $\Psi$ and $\Psi^{\dagger}$ represent the usual field operators, and $\langle\ldots\rangle_{H}$ denotes the averaging with respect to an arbitrary state of the full dynamical system (B1).

Employing the interaction picture Eq. (B2) can be transformed to

$$
i \mathcal{G}_{\alpha \beta}\left(t, t^{\prime}\right)=\left\langle T_{\mathcal{C}}\left[\widetilde{\Psi}_{\alpha}(t) \widetilde{\Psi}_{\beta}^{\dagger}\left(t^{\prime}\right) S_{\mathcal{C}}\right]\right\rangle_{H_{0}}
$$

with the contour scattering operator $S_{\mathcal{C}}$ defined by

$$
S_{\mathcal{C}}=T_{\mathcal{C}} e^{-\frac{i}{\hbar} \int_{\mathcal{C}} d t \widetilde{H}_{1}(t)} .
$$

The tilde in Eq. (B3) and (B4) characterizes the corresponding quantity in the interaction picture. Equation (B3) is suitable for performing the usual perturbation expansion in terms of $\widetilde{H}_{1}(t)$, the only difference being that all time integrals need to be taken over the time contour $\mathcal{C}$ instead of the real time axis.

Inspecting the definition of the contour ordered Green's function (B2) and the possible location of the two time arguments $t$ and $t^{\prime}$ on either the increasing $(+)$ or the decreasing $(-)$ branch of the contour we can decompose $\mathcal{G}_{\alpha \beta}\left(t, t^{\prime}\right)$ into the four analytical pieces

$$
\begin{aligned}
& i G_{\alpha \beta}^{++}\left(t, t^{\prime}\right)=\left\langle T_{c}\left[\Psi_{\alpha}(t) \Psi_{\beta}^{\dagger}\left(t^{\prime}\right)\right]\right\rangle_{H}, \\
& i G_{\alpha \beta}^{+-}\left(t, t^{\prime}\right)=-\left\langle\Psi_{\beta}^{\dagger}\left(t^{\prime}\right) \Psi_{\alpha}(t)\right\rangle_{H}, \\
& i G_{\alpha \beta}^{-+}\left(t, t^{\prime}\right)=\left\langle\Psi_{\alpha}(t) \Psi_{\beta}^{\dagger}\left(t^{\prime}\right)\right\rangle_{H}, \\
& i G_{\alpha \beta}^{--}\left(t, t^{\prime}\right)=\left\langle T_{a}\left[\Psi_{\alpha}(t) \Psi_{\beta}^{\dagger}\left(t^{\prime}\right)\right]\right\rangle_{H},
\end{aligned}
$$

where $T_{c}$ and $T_{a}$ denote the chronological and antichronological time ordering operator on the real time axis, respectively. Equation (B5) can be also expressed in matrix notation,

$$
\mathcal{G}_{\alpha \beta}=\left(\begin{array}{ll}
G_{\alpha \beta}^{++} & G_{\alpha \beta}^{+-} \\
G_{\alpha \beta}^{-+} & G_{\alpha \beta}^{--}
\end{array}\right),
$$

where the time arguments are omitted for convenience.

The time evolution of the Green's function (B6) is governed by the Dyson equation

$$
\mathcal{G}_{\alpha \beta}=\mathcal{G}_{\alpha \beta}^{(0)}+\mathcal{G}_{\alpha \delta}^{(0)} \Sigma_{\delta \gamma} \mathcal{G}_{\gamma \beta},
$$

where the summation over internal indices and integration over internal times is implicitly assumed. Equation (B7) involves the unperturbed Green's function (indicated by a (0) superscript) and the self-energy $\Sigma_{\delta \gamma}$. 
The latter is defined on the contour $\mathcal{C}$ as well and thus possesses a matrix representation similar to Eq. (B6). Hence, the Dyson equation (B7) is a matrix equation.

The structure of this equation can be simplified when it is noted that the set (B5) is linearly dependent. Applying the unitary transformation

$$
U=\frac{1}{\sqrt{2}}\left(\begin{array}{rr}
1 & -1 \\
1 & 1
\end{array}\right)
$$

to the Dyson equation (B7) the Green's function and the self-energy turn into

$$
\begin{gathered}
\widehat{\mathcal{G}}_{\alpha \beta}=U \mathcal{G}_{\alpha \beta} U^{\dagger}=\left(\begin{array}{cc}
0 & G_{\alpha \beta}^{A} \\
G_{\alpha \beta}^{R} & G_{\alpha \beta}^{K}
\end{array}\right), \\
\widehat{\Sigma}_{\alpha \beta}=U \Sigma_{\alpha \beta} U^{\dagger}=\left(\begin{array}{cc}
\Sigma_{\alpha \beta}^{K} & \Sigma_{\alpha \beta}^{R} \\
\Sigma_{\alpha \beta}^{A} & 0
\end{array}\right) .
\end{gathered}
$$

The superscripts $A, R$ and $K$ denote, respectively, the advanced, retarded and Keldysh part of the corresponding quantity. For the retarded and advanced Green's functions holds

$$
G_{\alpha \beta}^{A}\left(t_{1}, t_{2}\right)=\left[G_{\alpha \beta}^{R}\left(t_{2}, t_{1}\right)\right]^{*} .
$$

Carrying out the matrix multiplication in the transformed Dyson equation one obtains the set of equations that determines the different parts of the Green's function,

$$
\begin{aligned}
G_{\alpha \beta}^{A / R}= & G_{\alpha \beta}^{A / R(0)}+G_{\alpha \delta}^{A / R(0)} \Sigma_{\delta \gamma}^{A / R} G_{\gamma \beta}^{A / R}, \\
G_{\alpha \beta}^{K}= & G_{\alpha \beta}^{K(0)}+G_{\alpha \delta}^{K(0)} \Sigma_{\delta \gamma}^{A} G_{\gamma \beta}^{A} \\
& +G_{\alpha \delta}^{R(0)}\left[\Sigma_{\delta \gamma}^{K} G_{\gamma \beta}^{A}+\Sigma_{\delta \gamma}^{R} G_{\gamma \beta}^{K}\right] .
\end{aligned}
$$

Equation (B11b) can be solved iteratively to give the important relation

$$
\begin{aligned}
G_{\alpha \beta}^{K}= & {\left[\delta_{\alpha \gamma}+G_{\alpha \delta}^{R} \Sigma_{\delta \gamma}^{R}\right] G_{\gamma \xi}^{K(0)}\left[\Sigma_{\xi \nu}^{A} G_{\nu \beta}^{A}+\delta_{\xi \beta}\right] } \\
& +G_{\alpha \delta}^{R} \Sigma_{\delta \gamma}^{K} G_{\gamma \beta}^{A} .
\end{aligned}
$$

The Keldysh part of the Green's function can thus be computed from Eq. (B12) once the advanced and retarded parts are known. The diagonal component $G_{\alpha \alpha}^{K}$ can then be used to calculate the occupation of the state $\alpha$ at arbitrary times

$$
n_{\alpha}(t)=\frac{1}{2}\left[1-i G_{\alpha \alpha}^{K}(t, t)\right]
$$

* Contact:marbach@physik.uni-greifswald.de

1 W. Sesselmann, B. Woratschek, J. Küppers, G. Ertl, and H. Haberland, Phys. Rev. B 35, 1547 (1987).

2 Y. Harada, S. Masuda, and H. Ozaki, Chemical Reviews 97, 1897 (1997).

${ }^{3}$ L. N. Kantorovich, A. L. Shluger, P. V. Sushko, and A. M. Stoneham, Surface Science 444, 31 (2000).

${ }^{4}$ H. Winter, Physics Reports 367, 387 (2002).

${ }^{5}$ M. A. Lieberman and A. J. Lichtenberg, Principles of Plasma Discharges and Materials Processing (WileyInterscience, 2005).

6 P. Stracke, F. Wiegershaus, S. Krischok, and V. Kempter, Surface Science 396, 212 (1998).

7 N. Lorente, D. Teillet-Billy, and J.-P. Gauyacq, Surface Science 432, 155 (1999).

8 U. Kaldor, The Journal of Chemical Physics 81, 2406 (1984).

9 N. E. Christensen and B. Feuerbacher, Phys. Rev. B 10, 2349 (1974).

10 J. W. Gadzuk, Surface Science 6, 133 (1967).

11 B. Segall, Phys. Rev. 124, 1797 (1961).

12 K. Makoshi, Surface Science 254, 281 (1991).

${ }^{13}$ K. Makoshi and H. Kaji, Progress of Theoretical Physics Supplement 106, 327 (1991).

14 L. V. Keldysh, Sov. Phys. JETP 20, 1018 (1965).

15 A. Blandin, A. Nourtier, and D. W. Hone, J. Phys. France 37, 369 (1976).

16 P. Danielewicz, Annals of Physics 152, 239 (1984).

17 J. Rammer and H. Smith, Rev. Mod. Phys. 58, 323 (1986).
18 H. D. Hagstrum, Phys. Rev. 96, 336 (1954).

19 E. C. Goldberg and F. Flores, Phys. Rev. B 45, 8657 (1992).

20 H. Shao, D. C. Langreth, and P. Nordlander, Phys. Rev. B 49, 13929 (1994).

21 C. M. Dutta and P. Nordlander, Progress in Surface Science 67, 155 (2001).

22 M. A. Vicente Alvarez, V. H. Ponce, and E. C. Goldberg, Phys. Rev. B 57, 14919 (1998).

23 A. Yoshimori and K. Makoshi, Progress in Surface Science 21, 251 (1986).

24 D. R. Penn and P. Apell, Phys. Rev. B 41, 3303 (1990).

25 L. A. Salmi, Phys. Rev. B 46, 4180 (1992).

26 N. Bonini, G. P. Brivio, and M. I. Trioni, Phys. Rev. B 68, 035408 (2003).

27 M. A. Cazalilla, N. Lorente, R. D. Muiño, J.-P. Gauyacq, D. Teillet-Billy, and P. M. Echenique, Phys. Rev. B 58, 13991 (1998).

28 G. D. Mahan, Many Particle Physics (Physics of Solids and Liquids) (Springer, 2000).

29 G. Katz and R. Kosloff, The Journal of Chemical Physics 103, 9475 (1995).

30 B. Joulakian, J. Hanssen, R. D. Rivarola, and A. Motassim, Phys. Rev. A 54, 1473 (1996).

31 P. F. Weck, O. A. Fojón, B. Joulakian, C. R. Stia, J. Hanssen, and R. D. Rivarola, Phys. Rev. A 66, 012711 (2002).

32 G. L. Yudin, S. Chelkowski, and A. D. Bandrauk, Journal of Physics B: Atomic, Molecular and Optical Physics 39, 
L17 (2006).

33 R. J. Silbey, R. A. Alberty, and M. G. Bawendi, Physical Chemistry (Wiley, 2004).

${ }^{34}$ E. Clementi and C. Roetti, Atomic Data and Nuclear Data Tables 14, 177 (1974).

35 P. J. Jennings, R. O. Jones, and M. Weinert, Phys. Rev.
B 37, 6113 (1988).

36 D. Neilson, R. M. Nieminen, and J. Szymański, Phys. Rev. B 33, 1567 (1986).

37 N. W. Ashcroft and N. D. Mermin, Solid State Physics (Brooks Cole, 1976). 